\title{
eJRIEPS
}

Ejournal de la recherche sur l'intervention en éducation physique et sport

$28 \mid 2013$

Varia

\section{Principes pour l'étude de la tactique commune aux jeux sportifs collectifs et leur corrélation avec la préparation tactique des équipes et des joueurs}

Principles for to the study of common tactics in team sports and their correlation with the tactical preparation of teams and players

\section{Léon Teodorescu}

\section{OpenEdition}

Journals

Édition électronique

URL : http://journals.openedition.org/ejrieps/2934

DOI : $10.4000 /$ ejrieps.2934

ISSN : 2105-0821

Éditeur

ELLIADD

Référence électronique

Léon Teodorescu, «Principes pour l'étude de la tactique commune aux jeux sportifs collectifs et leur corrélation avec la préparation tactique des équipes et des joueurs ", eJRIEPS [En ligne], 28 | 2013, mis en ligne le 01 janvier 2013, consulté le 21 décembre 2020. URL : http://journals.openedition.org/ ejrieps/2934 ; DOI : https://doi.org/10.4000/ejrieps.2934

La revue eJRIEPS est mise à disposition selon les termes de la Creative Commons Attribution 4.0 International License. 
eJRIEPS 28 janvier 2013

Principes pour l'étude de la tactique commune aux jeux sportifs collectifs et leur corrélation avec la préparation tactique des équipes et des joueurs

\section{Léon Teodorescu}

Professeur des Universités

Chef de la Chaire de Jeux Sportifs de l'Institut de Culture Physique de Bucarest

Le colloque de Vichy en 1965 a beaucoup contribué à la réflexion sur les sports collectifs. Prononcée à cette occasion, la conférence du professeur Teodorescu visait à mettre en évidence des principes pour l'étude de la tactique commune aux jeux sportifs collectifs. Comme le texte de cette conférence est maintenant difficilement trouvable, j'ai donc proposé à eJRIEPS de publier, à nouveau, cet exposé. Ici, le professeur Teodorescu se consacre au fonctionnement interne de l'équipe à l'aide des analyses (techniques, tactiques....) très précises. Les organisations offensives et défensives sont caractérisées par "des bases, des principes, des facteurs" qui constituent un inventaire très complet de l'ensemble des éléments à prendre en compte pour le bon fonctionnement d'une équipe. Même si ce type d'inventaire reste assez formel, il concourt à une meilleure compréhension de la structure d'une équipe et des modalités de l'affrontement. L'autre contribution de cette approche par rapport au technicisme, a consisté à changer la conception d'une équipe. Le groupe de joueurs n'est plus la somme des individus qui la composent mais un ensemble structuré et dynamique en vue de la réalisation d'un projet commun. II doit exister une coordination réciproque des actions individuelles et collectives à partir de principes généraux pour mieux comprendre et organiser le jeu.

Bonne lecture.

Jean Francis Gréhaigne

Mots clés : sport d'équipe, technique, tactique, organisations défensives et offensives

A partir de l'expérience des spécialistes roumains dans le domaine des sports collectifs, nous avons choisi pour notre leçon le thème suivant: "Principes pour l'étude de la tactique commune aux jeux sportifs collectifs et leur liaison avec la préparation tactique des équipes et des joueurs ", compte tenu que la tactique est le facteur commun le plus évident lorsqu'on envisage en commun les jeux sportifs collectifs. 


\section{eJRIEPS 28 janvier 2013}

Evidemment, pour chaque étude, il est nécessaire de donner une définition autant que possible complète des termes respectifs, un contour autant exact des sphères des notions utilisées selon les raisonnements et observations faites, ainsi qu'un échelonnement de ces notions dans une succession logique du point de vue des rapports réciproques.

Etant donné que la littérature de spécialistes que nous avons consultée contient peu de données dans ce domaine - et parmi celles-ci ; beaucoup d'entre elles sont contradictoires -, notre leçon débutera par les questions de la terminologie fondamentale, générale pour tous les jeux sportifs collectifs, essayant ainsi s'exposer le contenu de chaque notion ainsi que leur rapport avec les autres notions. Nous allons commencer avec la notion de tactiques sur laquelle nous donnerons quelques considérations générales.

Nous affirmons que la tactique dans les jeux collectifs sportifs représente la contribution active du facteur conscience aussi bien pendant le match, qu'au cours de la préparation.

\section{Quelques définitions}

Pendant le match, la tactique constitue le moyen par lequel une équipe essaye de valoriser les qualités propres des joueurs, en créant des conditions pour cette mise en valeur et pour atténuer les défections dans leur préparation. Toujours à l'aide de la tactique, on poursuit l'annihilation des qualités positives des adversaires et la spéculation de leurs défauts. Par une tactique justement orientée, on cherche à obliger l'équipe adverse à entamer la lutte dans des conditions désavantageuses pour elle, et favorables pour sa propre équipe. Comme orientation générale, chaque équipe essaye de réaliser une confrontation directe des points forts des joueurs avec les points faibles des adversaires.

La tactique doit être considérée dans une unité dialectique avec la technique et les autres facteurs de l'entraînement (préparation physique et préparation psychologique), parmi lesquels la technique constitue le moyen principal à l'aide duquel se réalisent les tâches de la tactique.

La technique est un moyen par la raison. C'est la pensée qui donne un contenu à la tactique des procédés techniques, les transformant en actions. Pendant le match, la pensée - dénommée conventionnellement pensée tactique - a un caractère actif, favorisant la création. La pensée tactique agit d'autant plus que l'arsenal technique est plus riche.

La notion de "tactique " dans les jeux sportifs pourrait être définie comme suit : " La totalité des actions individuelles et collectives des joueurs d'une équipe, organisée et 


\section{eJRIEPS 28 janvier 2013}

coordonnée rationnellement et d'une façon unitaire - dans les limites du règlement du jeu et de l'éthique sportive- en vue d'obtenir la victoire ". La définition exposée correspond tant à la notion de tactique en général comme composante des jeux sportifs - à côté de la technique de préparation physique et psychologique - qu'à la notion de tactique collective d'équipe. Comme notions subordonnées à la notion de « tactique » nous mentionnons les suivantes :

1- L'attaque (l'offensive) : "situation tactique (la phase du jeu) quand une équipe se trouve dans la possession du ballon (ou d'un autre objet du jeu : ballon ovale, puck) et a la possibilité d'entreprendre des actions offensives ».

2- La défense : "situation tactique (la phase du jeu) quand une équipe lutte pour entrer en possession du ballon (pour s'en emparer) sans commettre des infractions sanctionnées par le règlement et sans permettre aux adversaires de réaliser un point ».

A côté de la tactique collective, il y a aussi une tactique individuelle qui correspond aux jeux sportifs, dénommée improprement par certains spécialistes « technique individuelle ». La technique sportive ne peut être qu'individuelle et elle a comme fondement la connaissance des procédés spécifiques de la manœuvre du ballon, ainsi que l'accomplissement de certains mouvements sans ballon. En échange, la tactique individuelle, ainsi qu'on l'a déjà exposé, suppose l'intervention du raisonnement dans l'utilisation des procédés techniques connus par les joueurs.

3- Pour définir la tactique individuelle, nous dirons que celle-ci représente «l'ensemble des actions individuelles utilisées consciemment par un joueur dans la lutte avec un ou plusieurs adversaires, tant dans l'attaque que dans la défensive ».

4- L'action individuelle : "l'utilisation consciente de la part d'un joueur -pendant une certaine phase d'attaque ou de défense - du complexe de procédés techniques les plus indiqués, au but de réaliser une tâche partielle (temporaire) du jeu; par exemple, le dépassement en dribbling d'un adversaire et le shoot au but (panier, etc...) ".

5- La combinaison tactique: "coordination des actions individuelles de deux ou plusieurs joueurs - dans une certaine phase du jeu- au but de réaliser une tâche partielle (temporaire) du jeu, par exemple, la plus simple combinaison d'attaque est la passe (le passage du ballon) ».

6- Le schéma tactique représente une forme plus évoluée et plus complexe de la combinaison tactique, étant en échange rigide, stéréotypé. On l'utilise d'habitude dans l'attaque, dans les phases obligatoires (les moments fixes) du match comme par exemple la remise du ballon dans le jeu en dehors du terrain. Essayant de donner une définition, 


\section{eJRIEPS 28 janvier 2013}

nous indiquons que le schéma tactique est : «la combinaison tactique exécutée d'un dispositif préétabli (établi à l'avance) dans lequel les joueurs et le ballon circulent et agissent stéréotypement, conformément aux indications établies à l'avance, afin de réaliser une tâche de moment de l'attaque ou de la défense ».

L'application des schémas tactiques aux moments décisifs du jeu provoque généralement la surprise chez l'adversaire et peut décider de la victoire.

7- La circulation tactique : celle-ci est appliquée de préférence dans l'attaque, servant comme phase de préparation collective de l'attaque finale. C'est une forme supérieure de schéma tactique. La circulation tactique s'applique dans l'ensemble du système du jeu. A son application participe un grand nombre de joueurs, groupés même en compartiments. Cette méthode est utilisée contre une défense déjà organisée, qui se trouve donc dans le dispositif. Nous essayerons de la définir ainsi : «la circulation tactique représente une suite d'actions individuelles et de combinaisons tactiques effectuées suivant un certain plan d'organisation, le ballon et les joueurs circulant successivement vers des endroits du terrain établis à l'avance ».

Par opposition au schéma tactique, la circulation tactique est élastique, supposant la détermination à l'avance non seulement de certains principes de déplacement (de circulation) du ballon et des joueurs, mais aussi le sens et le rythme de ces déplacements. Comme exemple de circulation tactique, nous citons le « huit » utilisé dans la pratique du basket-ball et du handball.

8- Le système du jeu représente la structure fondamentale de la tactique collective (d'équipe). A l'aide de ce système, on établit une différence entre les compartiments et les emplois (postes) dans l'équipe. A la majorité des jeux sportifs ainsi que le basket-ball, le handball, le polo, apparaissent des systèmes distincts pour l'attaque et pour la défense, tandis qu'aux autres jeux, tels que le football et le rugby, le système de jeu considère l'attaque et la défense en ensemble.

Tâchant de définir le système de jeu, nous pourrions dire qu'il représente : «la forme générale d'organisation des actions offensives ou défensives des joueurs, par l'établissement d'un dispositif précis, de certaines tâches - par emplois et compartiments ainsi que de certains principes de collaboration entre ceux-ci ». La sphère de la notion de système est plus petite que celle de la notion de tactique, étant subordonnée à la dernière, parce que la tactique d'une équipe peut consister en l'utilisation successive et temporaire de plusieurs systèmes de jeu, au cours du même match. 


\section{eJRIEPS 28 janvier 2013}

9- La conception du jeu : c'est une notion discutée concernant l'acceptation donnée à son contenu. Suivant notre avis, la conception du jeu représente : « les particularités ou caractéristiques que présente une équipe dans l'utilisation de la tactique. Ces particularités coïncident d'habitude avec la conception tactique de l'entraîneur ».

La conception du jeu est concrétisée par la sélection et la préparation de certains systèmes de jeu et des circulations tactiques qui constituent la tactique de base de l'équipe caractéristique dans tous les matchs.

10- Le plan tactique spécial : ce plan tactique spécial est élaboré pour chaque match suivant les particularités du jeu de l'équipe adverse. Ce plan est connu sous le nom de « stratégie »; sa caractéristique consiste en adaptations de la tactique de base de l'équipe (du système de jeu et de la circulation tactique, en un mot de la conception générale de jeu) aux particularités de l'adversaire. L'adaptation est faite, bien sûr, tenant compte aussi des particularités de l'équipe et des joueurs propres. Le plan, tactique spécial a un caractère temporaire et d'habitude son applicabilité reste valable uniquement pour le même adversaire et seulement dans certaines conditions données. En exprimant son contenu, nous montrons que le plan tactique spécial représente : «l'adaptation de la tactique de base de l'équipe au spécifique de l'adversaire par l'établissement de certaines tâches tactiques individuelles et collectives supplémentaires, ainsi que d'autres mesures et indications suivant lesquelles les joueurs développent leur jeu dans un certain match ». Les termes exposés jusqu'ici, sont des notions subordonnées à la tactique ; nous allons vous présenter plus loin seulement deux questions fondamentales, dont la sphère contient aussi la notion de tactique, donc y compris tous les termes décrits antérieurement.

11- Le style de jeu : celui-ci se réfère à l'équipe et ne doit pas être confondu avec le « style personnel » se référant aux particularités d'un joueur qui exécute la technique. Le style de jeu d'une équipe pourrait être défini aussi comme : « une personnalité collective » -si l'on peut dire ainsi. Ce fait ne se réalise pas chez toutes les équipes. Le style de jeu est le résultat d'un entraînement collectif de longue durée, auquel participe approximativement le même effectif, assez homogène, dans la conception du jeu, dans les qualités, ainsi que dans certains aspects principaux de la personnalité. Essayant de le définir, nous relevons que le style de jeu représente : « les caractéristiques en application de la technique, de la tactique et du rythme de jeu communs (ou généralités) des joueurs d'une équipe et manifestés dans les matchs ».

L'école par branches de jeux sportifs représente une notion plus ample, bien que dans l'acceptation courante elle est confondue avec le style de jeu. «L'école » représente : 


\section{eJRIEPS 28 janvier 2013}

« les caractéristiques dans la manière d'interprétation et d'application unitaire du même style de jeu et de la même conception de préparation sportive -correspondant à un grand nombre d'équipes sur un territoire- comme résultat de l'expérience et de la tradition sportives ; l'école est influencée par l'organisation sociale, par l'idéologie et parfois par certaines particularités spécifiques eu peuple respectif ». L'application des caractéristiques d'une école dépasse maintes fois le territoire d'un Etat, étant adoptée par les équipes de plusieurs Etats. II y a plusieurs « écoles » pour chaque jeu sportif ; pourtant leur nombre est relativement réduit. Ainsi, nous pourrions parler d'une «école française pour le rugby ", d'une "école américaine pour le basket ", d'une "école roumaine pour le handball » et d'une "école hongroise pour le football », etc... Ces écoles existent ensemble avec les écoles similaires anglaises, soviétiques, allemandes et autres écoles.

En conclusion à ce chapitre, nous voulons souligner que les termes présentés sont valables pour la tactique de tous les jeux sportifs, constituant un « genus proximum " pour les termes au caractère particulier spécifique à chaque jeu sportif. Pour ces raisons, nous considérons juste d'affirmer que ces termes sont considérés par nous comme des termes «fondamentaux » ou « de base ».

\section{Les bases de la tactique dans l'attaque et dans la défense}

Chaque jeu sportif consiste en une lutte permanente entre l'attaque (l'offensive) et la défense. L'avantage de l'attaque est l'initiative. Cette initiative est déterminée par la possession (la détention) du ballon, condition sine qua non pour la réalisation d'un point (goal). Le désavantage de l'attaque consiste dans les difficultés que présente la technique assez compliquée spécifique aux manœuvres du ballon, auxquelles s'ajoutent la nécessité de sa protection et son maintien contre les actions des défenseurs. Ceux-ci sont intéressés non seulement pour éviter le marquage d'un point, mais en même temps ils essayent d'entrer en possession du ballon. La défense est avantagée par le fait que la technique du jeu sans ballon n'est pas compliquée, et le nombre relativement réduit de procédés techniques en facilite l'appropriation, en échange, la défense présente le risque que chaque attaque de l'adversaire pourrait se terminer par le marquage d'un point, ce qui réalise un équilibre relatif entre l'attaque et la défense. La relation antagoniste entre l'attaque et la défense est manifestée individuellement (lutte entre attaquant et le défenseur) et collectivement (lutte entre l'attaque $t$ la défense). Chacun de ces deux agents du jeu (l'offensive et la défensive) cherche à rompre l'équilibre qui existe théoriquement- en se créant des avantages. Pour atteindre ce but il a été nécessaire de 


\section{eJRIEPS 28 janvier 2013}

trouver en dehors de l'appropriation des procédés techniques spécifiques à l'attaque et à la défense - les plus efficaces modalités d'utilisation de ces procédés, qui seraient organisés sous la forme d'actions individuelles et collectives.

L'organisation suppose un déroulement rationnel des actions et une coordination ; il a été donc nécessaire de trouver des principes, des règles, des formes ainsi que d'autres éléments convenables suivant lesquels le jeu devrait se dérouler, pour assurer le succès tant en attaque qu'en défensive. Les éléments de cette organisation rationnelle du déroulement du jeu constituent le contenu de la tactique.

Pour aboutir à la détermination de ce contenu, il a été d'abord nécessaire de faire une analyse et une étude du jeu, donc de la pratique. Continuant cette opération, on est passé à la synthèse, à la généralisation et à la systématisation de tout ce que la pratique de jeu avait de plus avantageux du point de vue de l'organisation et de la coordination des actions.

Excepté ceux-ci, on est arrivé par raisonnement à l'élaboration d'autres formes supplémentaires d'organisation, plus perfectionnées, dont l'efficacité a été confirmée après leur application dans la pratique du jeu. Par une collaboration continuelle entre la pratique et la théorie, on est arrivé au moment de la synthèse et de la systématisation des éléments fondamentaux de la tactique, connue sous le nom de "Bases de la tactique ". Ces éléments fondamentaux de la tactique, répartis entre l'attaque et la défense, constituent des paramètres valables pour l'étude de la tactique de n'importe quel jeu sportif collectif. L'étude de la tactique sur la base de ces paramètres offre les possibilités de trouver de nouvelles formes d'organisation des actions d'attaque et de défense, d'apprécier le plus judicieusement possible les caractéristiques du jeu des adversaires et de la propre équipe, pour assurer une préparation complète des joueurs. Envisageant les qualités des éléments fondamentaux de la tactique, ces derniers peuvent être considérés aussi comme des principes d'étude; le critère de base de l'efficacité des conclusions auxquelles on arrive est constitué par leur vérification permanente dans la pratique.

En généralisant les résultats de nos observations et ceux des autres spécialistes roumains, nous montrons que selon notre expérience les éléments fondamentaux de la tactique dans les jeux collectifs sont : les phases, les principes, les facteurs et les formes valables tant pour l'attaque que pour la défense.

Les phases représentent les étapes parcourues dans le déroulement tant de l'attaque que de la défense, dès leur initiation jusqu'à leur complète consommation. Nous mentionnons que, dans certaines situations, il n'est pas nécessaire de parcourir toutes les phases. 


\section{eJRIEPS 28 janvier 2013}

Les principes constituent les règles de base suivant lesquelles les joueurs dirigent et coordonnent leur activité -considérés individuellement et en collectif- durant les phases.

Les facteurs constituent les moyens de base par lesquelles les joueurs agissent dans les phases d'attaque ou de défense, en appliquant simultanément des principes.

Les formes représentent la structure organisatrice de l'activité des joueurs dans les diverses phases, en même temps que l'application des principes et l'utilisation des facteurs.

Nous allons maintenant concrétiser cette présentation abstraite des bases de la tactique des jeux sportifs collectifs, concernant l'attaque et la défense en commençant par :

2. 1. Les bases de la défense

Les phases de la défense sont les suivantes: l'équilibre défensif, la perte de la possession du ballon, l'arrêt de la contre-attaque, le repli, l'organisation du dispositif de défense et la défense proprement dite.

1- L’équilibre défensif : La défense organisée -caractéristique aux jeux modernescommence, bien que ceci paraisse paradoxal, en même temps que le déroulement de l'attaque. Elle consiste en mesures préventives prises par l'équipe attaquante, pendant la construction (la préparation) de l'action finale (shoot ou lancement au but ou au basket). L'équilibre défensif est assuré par plusieurs joueurs (d'habitude spécialisés comme défenseurs) qui sont placés et agissent derrière leurs propres attaquants engagés dans la lutte pour la rupture du dispositif de la défense adverse. En même temps, ils surveillent les joueurs adverses non engagés dans la lutte de défense. L'organisation de l'équilibre défensif a un triple but :

a) La réorganisation de la préparation de l'attaque en cas d'échec (donc but offensif)

b) La transition organisée dans la défense, après la perte de la possession du ballon

c) L'organisation d'une défensive temporaire, suivant la situation, jusqu'à l'encadrement de tous les défenseurs dans le dispositif de défense utilisé par l'équipe.

2- La perte de la possession du ballon: Cette perte est inévitable. Elle peut être classifiée comme suit : normale - au cas où l'équipe a réalisé un point ; plausible - si on a échoué au but pour des raisons tactiques, sauf réussir à réaliser un point ; anormale comme résultat de la violation du règlement ou d'une fausse manœuvre du ballon, ayant pour conséquence la prose du ballon par l'adversaire (passes interceptées, sortie du ballon hors du terrain de jeu, etc...).

Sans doute, il est préférable que la perte de la possession du ballon advienne seulement dans des conditions normales ou tout au plus plausibles. 


\section{eJRIEPS 28 janvier 2013}

3- L'arrêt de la contre-attaque : Cette phase consiste dans la lutte des attaquants pour entrer en possession du ballon, après sa perte près du but ou du basket adverse. Cette lutte a plus de succès dans le cas de certains jeux (football, rugby) et moins dans d'autres (basket, handball, polo) ; en échange, le résultat de cette lutte doit se solder par la détermination de l'adversaire -obligé de lutter pour le maintien du ballon- de tarder ou de renoncer au déclenchement rapide de la contre-attaque. Le temps gagné est utilisé en échange pour le repli et l'organisation de la défense.

4- Le repli ou la retraite de la défense : Celle-ci doit être exécutée d'une manière organisée et opportune. Elle commence en même temps que la perte de la possession du ballon et finit avec l'occupation du dispositif de défense.

L'organisation du repli consiste pour les défenseurs à appliquer le marquage sur le parcours des adversaires (des attaquants adverses) au cas où ceux-ci déploieraient la contre-attaque suite à l'échec des joueurs, qui ont essayé d'arrêter le lancement de la contre-attaque.

5- L'organisation du dispositif de défense : C'est, aussi comme le repliement, une phase critique où les défenseurs passent un examen de maturité tactique. Nous affirmons ceci, parce que, dans la plupart des cas, l'occupation des places du dispositif se passe en lutte directe avec les attaquants adverses, qui tentent de s'approcher davantage et le plus rapidement possible pour shooter. Si la préparation de l'équipe est faible, nous assistons d'habitude à une crise de placement dans le dispositif. Afin d'éviter une telle crise, il est préconisé que le placement dans le dispositif commence en même temps que le repliement. Au cours de l'occupation du dispositif, le principe de base de la défense est de ne pas recevoir un point (donc nettement destructif) et de gagner du temps pour l'organisation et, occasionnellement, on essaie d'entrer en possession du ballon. II faut ajouter que dans cette phase le principe de l'entraide a une grande importance.

6- La défense proprement dite constitue la phase principale de la défense. Elle suppose un dispositif organisé, une coordination et une collaboration dans les actions et essaie non seulement d'éviter l'écueil d'un point, mais aussi la prise rapide du ballon de l'adversaire et le passage à la contre-attaque. La lutte entre une attaque organisée et une défense organisée met en évidence la préparation et les qualités individuelles et collectives des joueurs et donne une note très spectaculaire, très goûtée par l'assistance.

2. 2. Les principes de la défense

Ceux-ci consistent en règle générale quant au comportement et à la coordination des actions des défenseurs afin de réaliser les objectifs de la défense. Les principes de la 


\section{eJRIEPS 28 janvier 2013}

défense sont les suivants; la défense du but (but, panier) ou l'empêchement des adversaires de réaliser un point: l'entrée en possession du ballon; l'adaptation de la défense au spécifique de l'attaque adverse et propre ; l'entraide (l'aide réciproque) par la détermination des attaquants de commettre des fautes et la mise en valeur de ces fautes.

1- La défense du but: Ce principe, le plus important, est connu sous le nom « d'empêchement de l'adversaire de marquer un but ». Si ce principe n'est pas respecté, cela mènerait inévitablement à la concession d'un point, compromettant le succès réalisé dans l'attaque, donc la perte du jeu. Indépendamment d'autres tâches, chaque joueur se trouvant à proximité de la zone périlleuse est obligé d'accorder la priorité à ce principe. L'application de ce principe -on pourrait dire vital- suppose toujours l'organisation du dispositif de défense, la répartition des tâches individuelles des défenseurs, l'organisation des compartiments et des règles de collaboration entre les joueurs et entre les compartiments. Les fautes dans l'organisation de la défense finissent toujours par des points reçus et donc, par la faillite dans la réalisation de la tâche primordiale de la défense, à savoir la consolidation des succès de l'attaque.

2- L'entrée en possession du ballon : Ce principe est caractéristique aux défenses modernes. Par son application, la défense cherche à priver l'initiative de l'attaque. Le succès de son application signifie un grand nombre d'attaques en faveur de sa propre équipe, donc des possibilités nombreuses de réaliser des points. L'application de ce principe suppose du dynamisme, de l'agressivité et de l'initiative. Par conséquent, la défense ne doit pas se borner simplement à des répliques données aux actions des adversaires, mais à des ripostes qui obligeront en permanence l'attaque adverse à se préoccuper de garder le plus longtemps possible la possession du ballon et de ne pas agir en priorité pour la réalisation d'un point. De là ressort le caractère agressif des défenses modernes.

3- L'adaptation de la défense au spécifique de l'attaque adverse et propre : La tactique, indifféremment de la situation, doit se caractériser par une élasticité, par une adaptation qui lui imprime un caractère opératif. L'adaptation au spécifique de l'attaque adverse signifie l'adoption de mesures convenables en vue de contrecarrer l'initiative et les qualités de l'attaque adverse, dues au système et aux combinaisons d'attaque, ainsi qu'aux particularités positives des joueurs qui les appliquent. Cette adaptation doit être réalisée compte tenu non seulement du principe de la défense du but, mais aussi celui à l'entrée en possession du ballon. En outre, la défense doit également être organisée en accord avec l'orientation de l'attaque de la propre équipe. L'application de cette dernière 


\section{eJRIEPS 28 janvier 2013}

indication est valable surtout pour le basket-balle, le handball, le polo et le volley-ball. L'adaptation de la défense spécifique de l'attaque propre suppose une telle disposition dans le dispositif de défense qui facilitera le déclenchement immédiat de la contre-attaque par les meilleurs joueurs, spécialisés en de telles actions. Pour exemplifier, nous relevons que les succès des équipes roumaines de handball sont dus en bonne partie à la stricte application de ce principe.

4- L'entraide : Indépendamment du système défensif adopté, la défense doit avoir un caractère collectif prononcé et permanent. Ce caractère est concrétisé par l'entraide organisée autour d'actions individuelles de défense. Si une défense individuelle est organisée et si cette action est disparate, elle ne pourra jamais faire face à une attaque collective. Par suite, chaque défenseur qui agit directement dans la phase de jeu doit être doublé. Cette doublure s'applique spontanément et en dehors des combinaisons de défense organisées, consacrées, par exemple, au changement d'adversaire. Un élément important, parmi d'autres, en application de l'entraide consiste à annoncer et à avertir de vive voix les intentions de défenseurs et des adversaires.

\section{5- La détermination des adversaires à commettre des fautes et la mise en valeur de} ces fautes: Ce principe est étroitement lié au principe de l'entrée en possession du ballon. Ceci suppose des actions correctes, opportunes et agressives, au moyen desquelles l'attaquant est déterminé à commettre des fautes sanctionnées par le règlement ou des fautes d'orientation pendant les actions offensives, ayant pour résultat l'interception du ballon. Ces fautes doivent être immédiatement mises en valeur, spécialement par une contre-attaque, surtout au handball, basket-ball et polo, quelle que soit la situation.

\section{3. Les facteurs de la défense}

Ils représentent les moyens principaux par lesquels agit le défenseur. Ils consistent en actions qui supposent $u$ processus de raisonnement, de sélection et de combinaison de procédés techniques spécifiques à la défense, en un arrangement correspondant à la phase concrète spontanée apparue dans le match. Nous avons donc à faire une opération d'analyse, de synthèse et de comparaison, qui précède, accompagne et poursuit les exécutions techniques. Au cours de telles actions, il est nécessaire d'appliquer les principes généraux et les règles spécifiques à la défense, en les adaptant en permanence avec la situation du terrain. Les facteurs principaux de la défense sont les suivants : le placement dans la défense, l'anticipation des actions, la circulation et les actions individuelles et collectives. 


\section{eJRIEPS 28 janvier 2013}

1- Le placement dans la défense consiste en l'occupation de la plus propice place du terrain, qui favorisera l'accomplissement des actions de la défense. Ce mouvement est exécuté tenant compte de son propre poste, du lieu de la position de l'adversaire et du secteur où se trouve le ballon, ainsi que du spécifique du système de défense utilisé par l'équipe.

2- L'anticipation des actions consiste dans la prévision des actions qui seront entreprises tant par les adversaires que par les membres de la propre équipe; il faudra agir d'une manière préventive, pour contrecarrer les premières et favoriser les autres.

3- La circulation représente généralement la distance parcourue par chaque défenseur en l'accomplissement des tâches de la défense. Cette circulation est organisée et appropriée par les défenseurs. La circulation doit avoir les caractéristiques suivantes :

Le maintien du placement correct par rapport au système et les particularités des adversaires

La circulation (les déplacements) d'un défenseur ne devra pas encombrer, mais au contraire, devra favoriser la circulation des autres joueurs de l'équipe

Elle doit favoriser l'exécution correcte et opportune des procédés de la défense.

Ces trois facteurs de la défense ont un caractère général et accompagnent les actions concrètes de défense, à savoir :

4- Les actions individuelles et collectives de défense, comme :

le marquage de l'adversaire

la dépossession du ballon

l'interception

les combinaisons tactiques entre les défenseurs.

Ces actions individuelles et collectives de défense ont un caractère concret et sont spécifiques comme terminologie et comme contenu à la tactique particulière de la défense de tout jeu sportif collectif.

5- Le rythme dans la défense consiste en des actions de défense plus ou moins nombreuses effectuées au cours d'une phase du jeu et par rapport à la durée de chaque action. La défense agressive comporte d'habitude un grand nombre d'actions, tandis que la défense passive en comporte un petit nombre; en ce cas on applique seulement le principe de la défense du but. 


\section{eJRIEPS 28 janvier 2013}

2. 4. Les formes de la défense

Elles consistent dans les divers systèmes de défense (le dispositif, les compartiments, les postes dans l'équipe et les principes de coordination de l'activité), dont nous nous sommes occupés au chapitre sur la terminologie.

En dehors des problèmes de structure supposés par le système de défense, les formes de la défense se rapportent aussi au caractère des actions de défense appliquées au cadre du système. De ce point de vue, il est à distinguer :

1- la forme active ou agressive, lorsque les défenseurs agissent en vue d'entrer en possession du ballon, cherchant à entraîner l'adversaire à commettre des fautes.

2- la forme passive lorsqu'on applique simplement le principe de la défense du but et que l'on attend les fautes de l'adversaire.

\section{Les bases de l'attaque}

Par opposition à la défense, l'attaque jouit de l'avantage de la possession du ballon, et, implicitement de l'initiative et de la capacité présumée de réaliser un point. La systématisation des éléments fondamentaux de l'attaque quant aux jeux sportifs collectifs est située dans le cadre du même tableau synoptique que nous avons exposé en parlant des "bases de la défense". Nous allons donc, tout en présentant les éléments constitutifs, nous borner à leur énonciation, avec quelques explications supplémentaires portant sur certains points spécifiques.

Nous voudrions rappeler que les bases de l'attaque sont toujours des : phases, principes, facteurs et formes.

3. 1. Les phases de l'attaque

1- L'entrée en possession du ballon qui est réalisée à la suite des actions défensives concrétisées par la dépossession de l'adversaire du ballon, les fautes réglementaires des attaquants adverses et la concession d'un point.

2- Le passage dans le terrain d'attaque. Cette phase apparaît lorsque la prise en possession du ballon a eu lieu dans la zone de défense. Ce passage doit être organisé d'avance, compte tenu que dans la plupart des cas, il advient pendant la lutte avec les adversaires. Par conséquent, cette action est préconisée avoir un caractère collectif. Les formes principales de l'organisation du passage dans le terrain d'attaque sont : la contreattaque, le passage rapide et le passage lent (individuel ou combinatif) 


\section{eJRIEPS 28 janvier 2013}

a) La contre-attaque est caractérisée par une grande vitesse un nombre réduit de passes et la supériorité numérique ou au moins un avantage positionnel. L'inscription d'un point par la contre-attaque détermine le renoncement aux autres phases de l'attaque.

b) Le passage rapide est organisé sur les principes de la circulation similaires à la contreattaque. La différence consiste dans la situation de la défense, qui n'est pas inférieure comme nombre, mais se trouve dans la phase de placement dans le dispositif, donc elle n'est pas encore organisée. Le dessein du passage rapide est celui d'assurer des conditions favorables de préparation et de finalité, avant que la défense adverse soit organisée.

c) Le passage lent est utilisé alors que les deux autres formes de passage à l'attaque ne sont pas indiquées. II faut retenir qu'au moment de ce commencement d'attaque la défense est déjà organisée dans le dispositif.

3- L'occupation du dispositif d'attaque suit le passage à l'attaque et suppose que les joueurs ont occupé leurs postes. Il est nécessaire d'insister aux entraînements sur l'importance d'une occupation rapide du dispositif, et sans faute dans le placement, afin qu'elle soit une continuation naturelle du passage à l'attaque.

4- La préparation de l'attaque est la phase fréquente de l'attaque. Elle consiste en des circulations et combinaisons tactiques, donc une question d'organisation. Cette phase poursuit une désorganisation de la défense adverse et la favorisation d'un des joueurs pour marquer un point.

5- La finalité de l'attaque consiste dans une action individuelle de la plus grande efficience et en même temps de responsabilité, concrétisée par le shoot, le lancement au but ou au panier, etc...

3. 2. Les principes de l'attaque

1- L'attaque du but adverse. L'application de ce principe présume l'orientation de toutes les actions vers le but adverse, donc de l'agressivité- et non stérilité et passivité des actions.

2- Le maintien de la possession du ballon. Ce principe est opposé aux tendances de risque irrationnel de certains joueurs, qui dissipent l'effort collectif des membres de l'équipe par des actions non conformes à la situation donnée. En cas d'échec de certaines combinaisons de préparation de l'attaque, la reprise de l'attaque est recommandée et en aucun cas l'aventure.

3- L'adaptation de l'attaque au spécifique de la défense adverse et aux particularités de la propre équipe. 


\section{eJRIEPS 28 janvier 2013}

\section{4- La détermination des défenseurs adverse à commettre des fautes et leur mise en} valeur.

Ces derniers principes ont été commentés au chapitre sur « les bases de la défense ».

3. 3. Les facteurs de l'attaque sont:

\section{1- Le placement en attaque ; 2- L'anticipation des actions}

Ces deux facteurs ont été également commentés au chapitre sur «les bases de la défense »

3- La circulation des joueurs. Bien que cette phase soit composée des mêmes éléments que la circulation des joueurs de la défense, son caractère dans l'attaque est beaucoup plus complexe et organisé. Le jeu sans ballon, concrétisé par des démarcations successives et simultanées constitue une des plus périlleuses armes de l'attaque moderne. La circulation des joueurs doit être liée de manière organique avec la circulation du ballon.

4- La circulation du ballon, qui est spécifique à l'attaque et consiste en passes et l'orientation du ballon. La circulation du ballon doit avoir un caractère offensif, elle doit protéger le ballon de l'intervention des défenseurs et favoriser son maintien ou la finalité de l'attaque.

5- L'organisation de l'attaque constitue la condition de réalisation de la phase de préparation de l'attaque. Elle consiste dans la coordination des actions individuelles et collectives.

6- Les actions individuelles et collectives sont le facteur principal de l'attaque. Elles consistent en des complexes de procédés techniques exécutés individuellement, dans la lutte avec l'adversaire et en collaboration avec les partenaires. Ces actions sont spécifiques pour chaque jeu sportif collectif, leur caractère général consiste seulement dans :

le démarrage individuel et avec l'aide du partenaire

le dépassement de l'adversaire par l'attaquant trouvé en possession du ballon la finalité

7- Le dépassement numérique est un facteur qui se réalise par des combinaisons tactiques et constitue un des buts immédiats de celles-ci. Par le dépassement numérique sont créées des conditions favorables pour l'action de finalité ou de maintien du ballon.

8- La surprise est un facteur qui résulte de l'ingéniosité des combinaisons et des actions tactiques. Par l'entremise de ce facteur, on vise à dissimuler les intentions et la détermination des défenseurs d'être en impossibilité d'appliquer le principe de 


\section{eJRIEPS 28 janvier 2013}

l'anticipation des actions. La surprise associée avec la supériorité numérique assure de bonnes conditions pour marquer.

9- L'assurance pourrait être interprétée aussi comme un principe de l'attaque et coïncide à la phase de la défense que nous avons nommée plus haut « équilibre défensif ». Cette phase a été analysée antérieurement.

10- La direction ou coordination de l'attaque. Le grand nombre d'actions, d'attaquants et de défenseurs nécessite l'intervention d'un coordinateur de l'attaque. C'est celui-ci qui adapte d'une manière créatrice le plan tactique de l'attaque par rapport aux conditions concrètes du terrain. Le dirigeant du jeu devra être caractérisé par un raisonnement tactique et technique, développé, par l'initiative, la clarté, l'expérience et l'autorité sur ses camarades d'équipe.

11- Le rythme. Son importance est beaucoup plus grande dans l'attaque que dans la défense et consiste dans le nombre plus grand ou plus réduit (du tempo) des actions individuelles et collectives de l'attaque et de la durée de chacune d'entre elles. Suivant le caractère des actions, le rythme peut être rapide ou lent. Chaque équipe a un rythme propre - le plus convenable. Pendant le match, la lutte se déroule en vue d'imposer le propre rythme à l'adversaire, c'est-à-dire de la déterminer à sortir de son rythme spécifique. Maintes fois, cette action est déterminante pour la victoire entre deux équipes d'une valeur relativement proche. Les jeux sportifs collectifs modernes sont caractérisés par des rythmes rapides, soutenus également par l'utilisation délibérée de variations de rythme, ce qui déroute l'adversaire.

\section{La corrélation entre les bases de l'attaque et de la défense avec l'entraînement tactique}

La détermination du contenu et de la systématisation des éléments fondamentaux de la tactique, conformément au rapport réel entre ces deux éléments, -rapport existant quant aux jeux sportifs de compétition- offre de multiples avantages pour les spécialités. II apparaît que le plus important de ces avantages est le fait qu'il offre une voie sûre pour l'apprentissage et le perfectionnement intégral de la tactique, c'est-à-dire de toutes les phases de l'attaque et de la défense. Combien de manuels de jeux sportifs collectifs analysent les phases de passage de la défense à l'attaque, celles d'occupation des dispositifs d'attaque ou de défense et de repliement? Tout cela, sans parler de la manière suivant laquelle devrait s'effectuer le passage rapide d'une phase à l'autre. 


\section{eJRIEPS 28 janvier 2013}

La plus grande partie de la littérature de spécialiste, ainsi que la pratique de l'entraînement sont axées en principal sur la phase de préparation de l'attaque et la mise en place de dispositif en défense. L'ignorance de l'importance des autres phases du jeu mène à une préparation incomplète des joueurs et leur activité dans l'exécution de ces phases est plutôt un effet de leur propre expérience, gagnée à l'aventure et moins que la suite d'un entraînement méthodique.

En conclusion, pour la pratique complète du jeu, nous considérons que des formes d'organisation des actions individuelles et collectives s'imposent, c'est-à-dire, une tactique pour toutes les phases de l'attaque et de la défense, ainsi que des exercices d'apprentissage et de perfectionnement de la tactique spécifique non seulement pour ces phases, mais également pour la corrélation entre les phases. Nous affirmons que les succès enregistrés par nos équipes de handball sont la conséquence d'une préparation méthodique par des exercices spécifiques. La préoccupation pour la préparation de toutes les phases du jeu ont influencé en grande mesure tant la technique que la tactique individuelle et collective, les complètent avec des aspects nouveaux, surtout ceux concernant la technique en vitesse et la continuation de la lutte organisée dans tous les secteurs du terrain et non seulement devant le but. Cette lutte organisée dans toutes les phases de l'attaque et de la défense a déterminé un accroissement et un accomplissement du caractère spectaculaire de certains jeux sportifs collectifs tels que le handball et le water-polo.

Enfin, comme dernières implications favorables importantes d'une étude complète des jeux sportifs collectifs réalisée sous l'aspect des phases, des principes, facteurs et formes de la tactique ainsi que par l'élaboration d'une terminologie unitaire et claire, nous mentionne :

1- Les possibilités de transfert de certaines actions tactiques d'un jeu sportif à l'autre ont augmenté.

2- on a créé des conditions pour le développement et même la révision de la méthode de l'entraînement dans les jeux sportifs collectifs, surtout concernant la préparation technique. Cette préparation technique n'est plus effectuée d'une façon isolée, mais dans des conditions plus proches du jeu, c'est-à-dire en lutte avec adversaire et en collaboration avec les membres de l'équipe; nous assistons donc à une préparation technique dans des conditions tactiques.

La préparation technique en conditions tactique a mené nécessairement à l'élaboration d'exercices ayant une nouvelle orientation, représentant des fragments d'actions tactiques 


\section{eJRIEPS 28 janvier 2013}

individuelles et collectives, spécifiques au système de jeu pratiqué par l'équipe. L'existence de ces fragments de jeu sous forme d'exercices a donné la possibilité d'extension de certains aspects de la " méthode de modélisation ", méthode de recherche scientifique réactualisée par la cybernétique et dans le domaine de l'entraînement sportif spécifique aux jeux sportifs collectifs. En vérité, une conception de jeu concrétisée par des systèmes, des circulations et des combinaisons tactiques, ainsi que par des exercices, en vue de leur appropriation par les sportifs, n'est au fond qu'un «modèle » élaboré théoriquement et vérifié ensuite par la pratique de la préparation des joueurs et de l'équipe. Ultérieurement, les modèles confirmés par la pratique entreront dans le patrimoine de la théorie et de la pratique de chaque jeu sportif.

\section{Conclusion}

Avec les dernières implications des considérations tactiques que nous avons présentées, nous relevons aussi celles concernant la planification de l'entraînement spécifique aux jeux sportifs collectifs. La modalité dont nous concevons la planification a des contingences avec l'emploi de certains aspects de la modélisation dont nous avons fait référence.

A notre avis, dans la planification, une constance devrait être le principe suivant lequel l'objectif détermine les tâches et que les principes déterminent les méthodes et leur caractère (la manière d'interprétation et les moyens d'application de ces méthodes). A la planification des entraînements spécifiques aux jeux sportifs collectifs, le point de départ devra être la conception tactique, c'est-à-dire le système de jeu et les circulations tactiques qui seront pratiquées par l'équipe en 1967, par exemple. Cette conception tactique constitue l'objectif et en même temps "le modèle théorique ". on établit ensuite, tenant compte des facteurs de l'entraînement, les procédés techniques, les actions tactiques individuelles et collectives et les niveaux de préparation physique, psychologique et théorique nécessaires pour la réalisation du jeu conformément à la conception, qui constitue l'objectif du plan de préparation de l'équipe respective. On compare ensuite le volume et la qualité de la préparation préconisée par la conception, avec le niveau de connaissances et la préparation des joueurs au moment où la préparation de perspective commence. On établit ensuite -toujours basés sur les facteurs de l'entraînement sportifquels éléments nouveaux devraient être appris, quels éléments devront être perfectionnés et quels éléments doivent être maintenus. Ces éléments seront ensuite répartis par années, périodes et étapes d'entraînement, tenant compte de leur influence réciproque ; 
eJRIEPS 28 janvier 2013

par exemple, l'apprentissage d'une combinaison tactique suppose l'apprentissage préalable de certaines actions tactiques et certains procédés techniques individuels. Le volume et la qualité des connaissances échelonnés pendant une année, une période ou une étape constituent les objectifs pour ces périodes de temps et en même temps des objectifs intermédiaires du plan intégral. On fixe certaines épreuves de contrôle nécessaires à la vérification de l'accomplissement des objectifs intermédiaires et l'entraîneur sélectionne ou imagine les exercices qu'il considère nécessaires pour la préparation proposée. 